\title{
Entangled plasmon generation in nonlinear spaser systems
}

\author{
Alexander Shesterikov ${ }^{1}$, Sergei Karpov ${ }^{1}$, and Mikhail Gubin ${ }^{2,1}$ \\ ${ }^{1}$ Vladimir State University named after A. G. and N. G. Stoletovs, Department of Physics and \\ Applied Mathematics, 600000 Vladimir, Russia \\ ${ }^{2}$ Moscow State Pedagogical University, Russian Institute for Advanced Studies, 119991 Moscow, \\ Russia
}

\begin{abstract}
The work is focused on the investigation of features of quantum dynamics for localized plasmons in spaser systems consisting of metal nanoparticles (NP) and semiconductor quantum dots (QDs). The nonclassical plasmon states generation in a three-particle spaser system with nonlinear plasmon-exciton interaction is predicted.
\end{abstract}

In this paper we propose a model of three nanoobjects (NP-QD-NP) coupled by nonlinear dipole-dipole interactions in the presence of an external magnetic field. The nonlinear regime of this ensemble corresponds to the two-quantum processes of the QD biexciton decay in the case $|\delta|>\Omega_{1,2},|\Delta|$, where $\delta=\varpi-\omega$ and $\Delta=\varpi-\omega_{p}, \varpi$ is the spasing frequency, and $\Omega_{1,2}$ are the Rabi frequencies of dipole-dipole interactions between the QD and NPs. As a result of this nonlinear process, one can expect the appearance of strongly correlated plasmon pairs. In the technical framework, the presented spaser systems can be used to generate nonclassical states of the electromagnetic field at the nanoscale. Such systems can be integrated in the individual plasmonic waveguides [1] and plasmonic circuits for quantum information processing [2]. Nonlinear regimes of interaction between NP and QD can be realized, firstly, in the presence of a two-photon pump in the system and secondly, under the condition that the coupling energy between two electron-hole pairs is of the same order of magnitude as the internal coupling energy of a single pair. The energy of biexciton state $X X$ differs from the double energy of the exciton $X$ by the biexciton binding energy $\Delta_{2 b}$ (see Fig. 1).
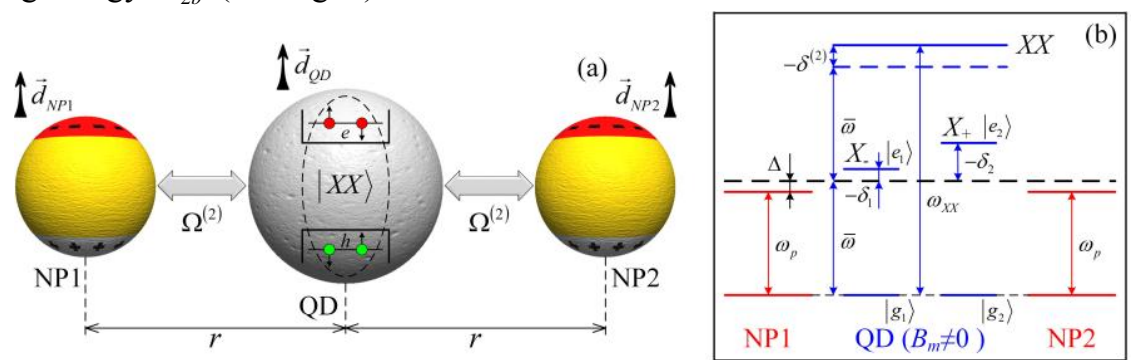

Fig. 1. (a) A model of a three-particle NP-QD-NP spaser. (b) The mapping of exciton and plasmon energy levels is in the presence of external magnetic field. 
The Hamiltonian of exciton-plasmon interaction has the following form [3]:

$$
H=h \omega_{p 1} \hat{c}_{1}^{+} \hat{c}_{1}+h \omega_{p 2} \hat{c}_{2}^{+} \hat{c}_{2}+h \omega_{X X} D / 2+h \Omega^{(2)}\left(\hat{c}_{1} \hat{c}_{2} \hat{S}^{+}+\hat{c}_{1}^{+} \hat{c}_{2}^{+} \hat{S}\right),
$$

where the last term in the brackets comprises the annihilation operator $\hat{S}$ of the biexciton $X X$ state and the creation operators $\hat{c}_{1}^{+}$and $\hat{c}_{2}^{+}$of a pair of plasmons, whose energies differ slightly for the different intermediate levels $X_{+}\left(X_{-}\right)$with frequencies $\omega_{X_{+}}\left(\omega_{X_{-}}\right)$. The parameter $\Omega^{(2)}=\Omega_{1} \Omega_{2} /(2|\delta|)$ is the effective two-quantum Rabi frequency, $D$ is the population imbalance. Parameters $\delta=\varpi-\omega_{X}$ and $\delta^{(2)}=2 \varpi-\omega_{X X}$ are the effective detunings, where $\omega_{X X}=\omega_{X_{-}}+\omega_{X_{+}}-\Delta_{2 b}$ is the biexciton frequency. The Rabi frequency of interaction between the QD and NP is $\Omega_{1}=\Omega_{2}=\mu_{Q D} / r^{3} \sqrt{\omega_{p} a_{N P}^{3} /\left(4 \pi h \varepsilon_{0}\right)}$, where $a_{N P}$ is the NP radius, $\omega_{p}$ is the plasmon resonance frequency of NPs, $\mu_{Q D}$ is the dipole moment. In this work we analyse the dynamics of the parameter

$$
G_{12}^{(2)}(t, \tau)=\frac{\left\langle\hat{c}_{1}^{+}(t) \hat{c}_{1}(t) \hat{c}_{2}^{+}(t+\tau) \hat{c}_{2}(t+\tau)\right\rangle}{\left\langle\hat{c}_{1}^{+}(t) \hat{c}_{1}(t)\right\rangle\left\langle\hat{c}_{2}^{+}(t+\tau) \hat{c}_{2}(t+\tau)\right\rangle},
$$

which corresponds to the cross-correlation function and is a criterion for establishing correlations between plasmonic modes $\hat{c}_{1}$ and $\hat{c}_{2}$. In particular, the condition $G_{12}^{(2)} \equiv G_{12}^{(2)}(t, 0)>1$ is associated with the intermode plasmon bunching. Moreover, the violation of the Cauchy-Schwarz inequality $C \equiv \frac{G_{12}^{(2)}(t, 0)^{2}}{g_{1}^{(2)}(t, 0) g_{2}^{(2)}(t, 0)} \leq 1$ indicates the nonclassical character of the correlations, where $g_{1(2)}^{(2)}=G_{1(2)}^{(2)}+1$. The maximum value of $C$ parameter is $C_{\max }=15.15$ and the stationary regime value is $C_{\mathrm{st}}=1.2$, which demonstrates the nonclassical character of correlations between plasmon modes in spaser system. Thus, the main result of our simulation is a demonstration of the development of quantum correlations between two localized plasmonic modes and the possibility for formation of an entangled state of plasmons. Further development of this work may be aimed on a complex simulation of nonlinear collective processes [4] with non-classical states of surface plasmon-polaritons (SPP) [5] and SPP waves structures [6].

This work was supported by the Russian Foundation for Basic Research Grant No. 17-42-330029 and Moscow State Pedagogical University Postdoctoral Fellowships in Humanities 2018, Grant No. 0-1067.

\section{References}

1. C. Reinhardt, A.B. Evlyukhin, W. Cheng et al., J. Opt. Soc. Am. B 30, 2898 (2013)

2. D.Yu. Fedyanin, D.I. Yakubovsky, R.V. Kirtaev, et al., Nano Lett. 16, 362 (2016)

3. M. Yu. Gubin, A. V. Shesterikov, S. N. Karpov, A. V. Prokhorov, Phys. Rev. B 97, 085431 (2018)

4. A.V. Golovanova, M.Yu. Gubin, M.G. Gladush, A.V. Prokhorov, Bull. Russ. Acad. Sci. Phys. 80, 808 (2016)

5. A.B. Shesterikov, M.Yu. Gubin, M.G. Gladush, A.V. Prokhorov, JETP 124, 18 (2017)

6. I.V. Dzedolik, V. Pereskokov, J. Phys.: Conf. Ser. 737, 012006 (2016) 